\title{
The clinical spectrum of primary Sjögren's syndrome: beyond exocrine glands
}

\author{
M.C. Leone, A. Alunno, G. Cafaro, V. Valentini, \\ E. Marcucci, E. Bartoloni, R. Gerli \\ Rheumatology Unit, Department of Medicine, University of Perugia, Italy
}

\begin{abstract}
SUMMARY
Although primary Sjögren's syndrome (pSS) is a mild indolent chronic disease mainly characterized by mucosal dryness in the majority of cases, a consistent subgroup of patients display extra-glandular manifestations. Virtually any organs and systems can be affected, leading to a more serious disease prognosis. Therefore, the prompt identification of patients at higher risk of extra-glandular manifestations is necessary to start a thorough follow up and an aggressive treatment. The aim of this review article is to provide an overview of epidemiological, clinical and serological features of extra-glandular manifestations in pSS as well as current knowledge about putative biomarkers useful in clinical practice.
\end{abstract}

Key words: Sjögren's syndrome; Extra-glandular manifestations; Cardiovascular risk.

Reumatismo, 2017; 69 (3): 93-100

\section{INTRODUCION}

Drimary Sjögren's syndrome (pSS) is a complex and heterogeneous chronic autoimmune disease primarily characterized by a focal lymphocytic chronic inflammation of exocrine glands leading to progressive loss of secretory function (1). Therefore, the specific case-history of pSS is dominated by signs and symptoms of mucosal dryness. However, although salivary and lachrymal glands represent the main target of pSS, several other organs and systems may be affected during the disease course in at least one third of patients, resulting in a plethora of systemic clinical manifestations, serological abnormalities and complications (2). The most severe complication of pSS is non-Hodgkin lymphoma occurring in about $5 \%$ of patients (3) and dramatically worsening disease prognosis. In this regard, the prompt identification of pSS patients with higher risk of developing extraglandular manifestations is mandatory for correct management. Low C3 and C4 levels, cryoglobulins, monoclonal component, anti-Ro/ SSA, anti-La/SSB, rheumatoid factor and hypergammaglobulinaemia represent poor prognostic serological factors in pSS associated with lymphoma and severe extraglan- dular features (2). Furthermore, histological features such as the presence of germinal centre-like structures in the minor salivary glands have been associated with higher risk of developing lymphoma (4).

The purpose of this review article is to discuss current knowledge of extra-glandular manifestations in pSS not secondary to mucosal dryness, excluding pathogenesis and clinical manifestations of lymphoproliferative disorders.

\section{MUSCULOSKELETAL INVOLVEMENT}

Musculoskeletal manifestations such as myalgia, arthralgia and morning stiffness are present in as many as $90 \%$ of patients, while clinically evident arthritis is found in up to $17 \%$. In a recent study conducted on 17 female patients with pSS and 18 patients with secondary SS (sSS), ultrasonography (US) was used to confirm the non-erosive nature of joint involvement in pSS and to discriminate between pSS and RA-associated SS. Synovitis was commonly detected in pSS, mainly in metacarpophalangeal joints (MCP), wrists, and knees. Bone erosions also may occur. Power Doppler signal as well as bone erosions could be rarely detect-
Indirizzo per la corrispondenza:

Roberto Gerli

Rheumatology Unit,

Department of Medicine

University of Perugia,

Edificio C, $5^{\circ}$ Piano

Piazzale Menghini, 1

06129 S. Andrea delle Fratte (PG), Italy

E-mail: roberto.gerli@unipg.it 
ed and the latter represented the better performing US feature to discriminate between pSS and RA-associated SS (5).

Muscle involvement is relatively frequent as diffuse pain and/or muscular weakness. Myalgia has been reported in $33 \%$ of patients although in about $47-55 \%$ of cases a possible overlap with fibromyalgia has been described $(6,7)$. Indeed, the prevalence of fibromyalgia in pSS is about $30 \%$ (8). Myositis has been reported in $3 \%$ of patients. Nonetheless, $5 \%$ to $73 \%$ of patients may reveal subclinical histopathological myositis (9-11). Muscle biopsy, the gold standard for the diagnosis of inflammatory myopathies, generally shows a perivascular inflammation or an interstitial myositis without involvement of muscle fibres. Although muscular involvement is usually detected over the course of pSS (10), in some cases it may also appear at disease onset. A recent study reported the prevalence of myositis in an Italian multicenter cohort of $1,320 \mathrm{pSS}$ patients. Among these, 17 patients presented signs and symptoms suggestive for IM; however only 6 cases had histologically confirmed myositis and, according to Bohan and Peter's diagnostic criteria, only 10 patients fulfilled at least the 3 required criteria for a probable diagnosis of myositis (12). Overall, a good response to immunosuppressive agents was observed with a good prognosis. Of interest, Espitia-Thibault et al. described a peculiar histological pattern in the muscle biopsy of 4 patients with pSS with germinal centre-like structures resembling those of minor salivary glands (13). In these patients, the clinical presentation was non-specific and non-severe with myalgias, muscular weakness and normal or elevated $\mathrm{CK}$ values.

\section{CUTANEOUS INVOLVEMENT}

The most prevalent skin findings in pSS include xerosis, purpura associated with vascular or hematologic abnormalities, Raynaud's phenomenon, annular erythema (AE) and other manifestations related to diseaseinduced cutaneous dryness (i.e., eyelid dermatitis and angular cheilitis). AE is an erythematous, photosensitive rash that appears as annular polycyclic lesions characterized by a wide elevated border and central pallor and may result in hypopigmentation. The histopathological hallmark is represented by perivascular lymphocytic infiltration. $\mathrm{AE}$ is mainly localized on the face, upper arms, neck and may be the first manifestation of the disease. The dramatically higher prevalence in Asian patients suggested a possible effect of ethnic background. Three clinical types of AE have been described in Japanese patients: type I is an isolated doughnutring-like erythema with an elevated border, type II resembles subacute cutaneous lupus (SCLE)-like marginally scaled polycyclic erythema and type III is a papular insect bitelike erythema (14). A recent retrospective study including 377 Spanish pSS patients revealed that $\mathrm{AE}$ is not an exclusive cutaneous feature of Asian patients with pSS. Indeed, $9 \%$ of Spanish pSS patients were diagnosed with $\mathrm{AE}$ and in over $70 \%$ this was before the diagnosis of pSS (15).

Vasculitis can affect different organs and systems, with the skin being the most frequent site. Hypergammaglobulinemic vasculitis (HGV) is a cutaneous vasculitis, related to a benign B-cell proliferation, whereas cryoglobulinemic vasculitis (CV) is a systemic immune complex-mediated vasculitis with complement activation and a higher risk of lymphoma. It is therefore required to differentiate the two forms in pSS patients with purpura. As observed by Quartuccio et al. in a large Italian cohort of pSS patients, peripheral neuropathy, low $\mathrm{C} 4$, leucopaenia, serum monoclonal component and the presence of anti-SSB/La antibodies characterized CV whereas rheumatoid factor positivity, leukopenia, serum monoclonal component, antiSSA/Ro antibodies and lymphoma were significantly associated with HGV (16).

The detection of ANCA positivity in pSS patients does not necessarily reflect an underlying ANCA-associated vasculitis (AAV) or single-organ AAV (17). However, several cases of coexisting pSS and AAV have been described (18).

\section{PULMONARY INVOLVEMENT}

The prevalence of pulmonary involvement in patients with $\mathrm{pSS}$ is estimated at between 
9 and $24 \%(19,20)$. However, evaluation of asymptomatic patients, with pulmonary function tests (PFTs), bronchoalveolar lavage (BAL), and computed tomography allows detection of abnormalities in up to $75 \%$ of patients $(21,22)$. Pulmonary manifestations in pSS typically affect the small airways, follicular bronchiolitis being the most common histological finding. However, any other component of the respiratory system may be affected. Nasal crusting with epistaxis, hoarseness and dry cough may occur because of mucosal dryness and thick mucus coating the vocal cords. Pleural effusion, sometimes associated with pericardial effusion, represents a common extraglandular manifestation in pSS. Interstitial lung disease (ILD) may occur in $3-11 \%$ of pSS patients (21) with several histopathologic patterns, including nonspecific interstitial pneumonia (NSIP), usual interstitial pneumonia (UIP), organizing pneumonia (OP), lymphocytic interstitial pneumonia (LIP) and cryptogenic organizing pneumonia (COP). NSIP is the most commonly observed histopathological pattern in ILD associated with connective tissue diseases, including pSS. LIP has a typical radiographic appearance of ground glass opacities with thin-walled cysts, and the presence of these cysts on a HRCT scan should raise clinical suspicion for $\mathrm{pSS}$ with ILD. Diffuse ILD is the most serious form of lung involvement due to its potentially progressive nature and simultaneously its risk of respiratory failure. The presence of low DLCO has been associated with higher mortality rates. Recently, an estimated prevalence of $22 \%$ of diffuse ILD in pSS in a population-based hospital patient cohort in Oslo has been described by Palm et al. Patients with lung involvement had reduced quality of life (QoL) and increased mortality compared to pSS patients without this manifestation (19). In the study from Reina et al., 25 patients with pSS-ILD were more likely to be positive for antiSSA antibodies. Nevertheless, in $60 \%$ of the patients, the diagnosis of ILD preceded that of pSS (23). Conversely, Roca et al. reported that only 5 (24\%) out of 21 patients were diagnosed with ILD prior to the di- agnosis of pSS (24). The histopathological patterns observed were NSIP, UIP, OP and LIP (in this order) and the majority of ILD patients had an acute/subacute onset (24). Overall, in $84 \%$ of patients pSS-ILD was either stable over time or worsening, while older age was associated with ILD deterioration. Therefore higher rates of mortality and morbidity in PSS are most likely related to ILD (23).

Amyloidosis, granulomatous lung disease, pulmonary lymphoma, cystic lung disease, pseudolymphoma and pulmonary arterial hypertension have been also described but with lower frequency in $\mathrm{pSS}$.

\section{RENAL INVOLVEMENT}

Renal disease can occur either as epithelial disease with a predominantly mononuclear lymphocytic infiltration resulting in tubulointerstitial nephritis (TIN) or as non-epithelial disease with a secondary immune complex-mediated process resulting in glomerulopathy. Overall, renal involvement occurs in $4-5 \%$ of pSS patients (25-27). TIN is the predominant condition, found in $75 \%$ of patients, with the remaining $25 \%$ of patients having glomerular disease (28, 29). In more detail, proximal injury was observed in $10-42 \%$, distal renal tubular acidosis in 5-24\% and a concentrating defect in $17-28 \%$.

Type II proximal tubular dysfunction consists of proteinuria, aminoaciduria, glycosuria, phosphaturia, uricosuria and bicarbonaturia (Fanconi syndrome) and may lead to osteomalacia as a consequence of phosphate wasting. Type I and IV distal renal tubular acidosis is due to a complete or incomplete inadequate $\mathrm{H}+$ secretion in the cortical collecting duct by the acid-secreting a-intercalated cells (30). Distal renal tubular acidosis in pSS was found to be associated with anti- Ro and La antibodies, longer disease duration, xerostomia, hypertension, higher creatinine and proteinuria, and also hypergammaglobulinaemia (31). pSS patients may also show hypokalaemic symptoms, including paralysis (32), nephrolithiasis or nephrocalcinosis. Nephrogenic diabetes insipidus and other acquired 
tubular defects affecting other tubular segments may also occur, the latter causing acquired Bartter or Gitelman-like syndromes. Glomerular involvement is uncommon and late in the course of pSS. It is usually associated with mixed cryoglobulinaemia and proliferative glomerulonephritis on kidney biopsy (27). The majority of glomerular diseases reported in pSS is represented by immune complex-mediated conditions, usually with the characteristic mesangioproliferative glomerulonephritis (MPGN), which is the most common glomerular lesion in pSS and is caused by the deposition of immune complexes, often cryoglobulins. MPGN may occur either alone or as part of a systemic vasculitis. It is also associated with lymphoma development and thus increased morbidity and mortality (27). Shavit et al. emphasised that patients with pSS can show atypical kidney pathology such as pauci-immune necrotising crescentic ANCA-related glomerulonephritis. ANCA positivity in SS might be associated with a higher burden of extraglandular manifestations, and a possible tendency to develop pauci immune glomerulonephritis (33).

\section{PERIPHERAL AND CENTRAL NERVOUS SYSTEM INVOLVEMENT}

Neurological manifestations have a frequency ranging from 10 to $60 \%$ and they may appear before other signs and diagnosis of pSS (34). The involvement of the peripheral nervous system (PNS) is more common and dominant at the upper limbs and the trunk. It may appear in different forms: sensorymotor polyneuropathy, pure sensory neuropathy (peculiar to $\mathrm{pSS}$ ), neuronopathy (related to lymphocytic infiltration of the dorsal root ganglia), autonomic neuropathy, cranial nerve involvement (mainly the cochleovestibular nerve but also the trigeminal and facial nerves), mononeuritis multiplex, chronic polyradiculoneuropathy, motor neuron disease or small fiber neuropathy (diagnosed on skin biopsy). Symmetrical axonal sensorimotor polyneuropathy is the most common type of polyneuropathy and is associated with higher monoclonal B-cell proliferation markers (hypergammaglobulinemia cryoglobulinemia, monoclonal gammopathy, serum free light chain ratio, and B-cell lymphoma) (35). Pure sensitive neuropathy (PSN) mainly occurs in females with a younger age at pSS diagnosis and fewer immunological markers. PSN has a chronic and insidious onset with a poor response to treatment with corticosteroids or immunosuppressive agents. Conversely, multiplex mononeuropathy is strongly associated with underlying vasculitis and high prevalence of severe parotid involvement, extraglandular features, cytopenias and immunological markers, consistent with a high systemic activity. Therefore, although this PNS manifestation displays excellent therapeutic response to corticosteroids and immunosuppressive agents, patients have a bad prognosis and low survival rate, due to the underlying systemic vasculitis (36). Sensorimotor neuropathy or mononeuritis multiplex mainly result from immunovascular injury, as indicated by the association with Raynaud's phenomenon, cutaneous vasculitis, and renal involvement. Conversely, sensory neuropathies are more probably linked to lymphocytic infiltration rather than to cryoglobulinemia and have almost no extraglandular involvement. Finally, as far as the parasympathetic cholinergic system is concerned, recent data demonstrated that the dysfunction of this system observed in pSS appears to be independent of gland inflammation and atrophy (37).

Central nervous system (CNS) involvement in $\mathrm{pSS}$ is less frequent than PNS involvement and is characterized by heterogeneous manifestations like stroke associated with CNS vasculitis, seizure, parkinsonism, chorea, central pontine myelinosis, cerebellar ataxia, encephalopathy, aseptic meningoencephalitis, transverse myelitis, chronic progressive myelitis, optic neuropathy, neuromyelitis optica, multiple sclerosis-like relapsing-remitting syndromes (34). High variable prevalence of CNS manifestations ranging from 2.5 and $60 \%$ of all pSS patients has been reported.

Most common symptoms are headache (46.9\%), followed by cognitive $(44.4 \%)$ and mood disorders (38.3\%). Seizures are 
observed in $20 \%$ of cases, pyramidal signs in $10 \%$ of cases, cerebellar signs in $10 \%$ of cases, neuromyelitis optica and meningoencephalitis in less than $5 \%$ of cases (38).

Headache, cognitive disorders and psychiatric symptoms appear to be associated with anti-SSA antibodies and Raynaud's phenomenon, the latter suggesting a possible underlying endothelial dysfunction (autoimmune endothelitis) of the cerebral microcirculation or a potential inflammation-mediated shift of the neurovascular coupling (38). Interestingly, abnormalities in the electrophysiological parameters can also be detected in asymptomatic patients with longer disease duration, salivary gland ultrasonography abnormalities and elevated inflammatory markers in blood tests (39).

\section{SWALLOWING DISORDERS, GASTROINTESTINAL AND LIVER MANIFESTATIONS}

Swallowing disorders are relatively common in pSS and increase with disease severity (40). Both oropharyngeal and esophageal swallowing disorders have been reported in this population, often attributed to sicca of the mouth and throat, loss of dentition, cricoarytenoid joint arthritis, neuropathy, esophageal abnormalities, and gastroesophageal reflux. The prevalence of swallowing disorders in pSS is over $60 \%$ and risk factors were represented by laryngeal irritants, such as secondary exposure to tobacco smoke, post nasal drip, frequent sinus infections, esophageal reflux, and acidic foods which may be related to reflux (41). Several other risk factors included neck/throat tension, voice problems lasting longer than 4 weeks, which seem to have anatomical connections to structures involved in swallowing. Chronic, longstanding swallowing disorders are common in pSS and consistently affect the patients' quality of life. The lack of salivary secretion may account for a reduced capacity to balance the gastric reflux $\mathrm{pH}$ leading to heartburn. On the other hand, reduced acid production has been also observed in pSS. Dyspepsia without major endoscopic alterations has been reported in about $20 \%$ of pSS patients. An infection supported by Helico- bacter pylori should always be treated as it represents a risk factor for the development of gastric lymphoma.

The liver is mainly involved due to associated conditions such as autoimmune hepatitis or primary biliary cirrhosis. Abnormal liver function tests can be detected in up to $50 \%$ of patients but usually they are mild and not associated with any organ-specific condition (42).

\section{CARDIOVASCULAR MANIFESTATIONS}

Heart involvement has been scarcely reported in pSS. However, studies based on echocardiography revealed a consistent prevalence of valvular disease, with mild mitral and aortic regurgitation being the most prevalent conditions, and of pulmonary hypertension. Sub-clinical pericardial effusion can also be detected in a small proportion of patients $(43,44)$. Importantly, pSS patients with anti-Ro and anti-La antibodies wishing to become pregnant should be informed about the risk of congenital heart block (CHB) (45). CHB is characterized by autoimmune-mediated damage to the fetal heart conduction system and in the majority of cases results in irreversible complete atrioventricular block. Unfortunately, preventive strategies are lacking, hence CHB is still a severe life-threatening disorder. Altered immune system function and inflammatory factors accelerate both the initiation and the progression of atherosclerosis (ATS) in patients with systemic rheumatic diseases, in particular RA and systemic lupus erythematosus. With regard to pSS, increasing evidence confirms that higher cardiovascular (CV) ATS is a severe complication also in this disease. Endothelial dysfunction is the first stage of subclinical ATS, promoted by an altered balance between endothelial microparticles (EMPs) released by damaged endothelium and endothelial progenitor cells (EPCs) compromising reparative capacity. We recently observed that pSS patients display increased proportions of both EMPs and EPCs with respect to normal subjects (46). These data underline a condition of chronic persistent endothelial fragmentation 
characterizing pSS patients independently of disease clinical activity, inflammatory background or disease severity.

Sub-clinical CV involvement can be assessed through plasma asymmetric dimethylarginine (ADMA) concentration, coronary flow reserve (CFR), intima media thickness (cIMT), pulse wave velocity (PWV) and myocardial deformation. In pSS ADMA, right and left PWV and left cIMT resulted higher, whereas CFR resulted lower in comparison with normal subjects. In addition, despite left ventricular systolic and diastolic function being similar in pSS and controls, echocardiographic findings support the presence of myocardial alteration in the former (47-50). Interestingly, not only the Framingham Risk Score (FRS) but also the SS damage index (SSDI) were independently associated with PWV (48). In this regard, an increased subclinical atherosclerosis is observed only in pSS patients with a longer disease duration, when the chronic inflammation might have caused a stabilized arterial wall damage. Furthermore, advanced age, higher focus score on minor salivary gland biopsy and reduced salivary flow rates, the presence of Raynaud's syndrome and reduced lymphocyte numbers were predictors of plaque formation (50).

Two recent large epidemiological studies attempted to characterize the prevalence of traditional $\mathrm{CV}$ risk factors and $\mathrm{CV}$ events in pSS patients $(51,52)$. Hypertension was the most prevalent $\mathrm{CV}$ disease risk factor, followed by hypercholesterolaemia, and hypertriglyceridemia. Consequently a higher proportion of $\mathrm{pSS}$ patients were taking antihypertensive and statin therapies in comparison with normal subjects. Conversely, smoking, obesity and diabetes mellitus were less prevalent in pSS than in controls. At least one CV event occurred in about $5 \%$ of patients, cerebrovascular events being the most common CV manifestation, followed by heart failure, angina and myocardial infarction. Older age, longer disease duration and the co-occurrence of severe extra-glandular manifestations were significantly associated with an increased prevalence of $\mathrm{CV}$ events. With re- gard to metabolic syndrome (MetS), this also resulted more prevalent in pSS patients compared with controls and associated with an abnormal adipokine profile. The relationship between MetS and IL-1 $\beta$ suggests the role of inflammation in the pathogenesis of this condition. On the other hand, no association between MetS and disease duration, extra-glandular involvement or prednisone use was observed (53).

\section{HAEMATOLOGICAL MANIFESTATIONS}

Chronic anemia and hypergammaglobulinemia are the most prevalent hematologic manifestations encountered at diagnosis and during the course of pSS. Leukopenia can also occur in about $20 \%$ of patients $(54,55)$. Only a few studies have been conducted on hematologic abnormalities and the significance of cytopenia in pSS is often disregarded in daily practice because its degree is usually mild and asymptomatic. Interestingly, however, any cytopenia has been associated with severe ocular surface damage even in patients with few ocular symptoms, while leukopenia with cardiovascular manifestations such as angina and neutropenia has been associated with a higher risk of lymphoproliferation (56). With regard to hypergammaglobulinemia and cryoglobulinemia, their association with higher risk of lymphoproliferation is now well established $(16,57)$.

\section{CONCLUSIONS}

The clinical picture of pSS is pleomorphic, ranging from mild sicca symptoms to severe life-threatening involvement of vital organs. It is therefore crucial to recognize patients at higher risk of extra-glandular manifestations and of $\mathrm{CV}$ events to ensure prompt preventive strategies and aggressive treatment when required. The identification of reliable biomarkers allowing the stratifying of patients with different disease evolution will ensure an optimal management of patients with this disease.

Conflict of interests: nothing to disclose. 


\section{REFERENCES}

1. Brito-Zerón P, Baldini C, Bootsma $\mathrm{H}$, et al. Sjögren syndrome. Nat Rev Dis Primers. 2016; 2: 16047.

2. Baldini C, Pepe P, Quartuccio L, et al. Primary Sjögren's syndrome as a multi-organ disease: impact of the serological profile on the clinical presentation of the disease in a large cohort of Italian patients. Rheumatology (Oxford). 2014; 53: 839-44.

3. Liang Y, Yang Z, Qin B, Zhong R. Primary Sjögren's syndrome and malignancy risk: a systematic review and meta-analysis. Ann Rheum Dis. 2014; 73: 1151-6.

4. Theander E, Vasaitis L, Baecklund E, et al. Lymphoid organisation in labial salivary gland biopsies is a possible predictor for the development of malignant lymphoma in primary Sjögren's syndrome. Ann Rheum Dis. 2011; 70: 1363-8.

5. Amezcua-Guerra LM, Hofmann F, Vargas A, et al. Joint involvement in primary Sjögren's syndrome: an ultrasound "target area approach to arthritis". Biomed Res Int. 2013; 2013: 640265.

6. Iannuccelli C, Spinelli FR, Guzzo MP, et al. Fatigue and widespread pain in systemic lupus erythematosus and Sjögren's syndrome: symptoms of the inflammatory disease or associated fibromyalgia? Clin Exp Rheumatol 2012; 30: 117-21.

7. Priori R, Iannuccelli C, Alessandri C, et al. Fatigue in Sjögren's syndrome: relationship with fibromyalgia, clinical and biologic features. Clin Exp Rheumatol. 2010; 28: S82-6.

8. Choi BY, Oh HJ, Lee YJ, Song YW. Prevalence and clinical impact of fibromyalgia in patients with primary Sjögren's syndrome. Clin Exp Rheumatol. 2016; 34: S9-13.

9. Kraus A, Cifuentes M, Villa AR, et al. Myositis in primary Sjögren's syndrome. Report of 3 cases. J Rheumatol. 1994; 21: 649-53.

10. Lindvall B, Bengtsson A, Ernerudh J, Eriksson $\mathrm{P}$. Subclinical myositis is common in primary Sjögren's syndrome and is not related to muscle pain. J Rheumatol. 2002; 29: 717-25.

11. Vrethem M, Lindvall B, Holmgren H, et al. Neuropathy and myopathy in primary Sjögren's syndrome: neurophysiological, immunological and muscle biopsy results. Acta Neurol Scand. 1990; 82: 126-31.

12. Colafrancesco S, Priori R, Gattamelata A, et al. Myositis in primary Sjögren's syndrome: data from a multicentre cohort. Clin Exp Rheumatol. 2015; 33: 457-64.

13. Espitia-Thibault A, Masseau A, Néel A, et al. Sjögren's syndrome-associated myositis with germinal centre-like structures. Autoimmun Rev. 2017; 16: 154-8.

14. Katayama I, Kotobuki Y, Kiyohara E, Murota H. Annular erythema associated with Sjögren's syndrome: review of the literature on the management and clinical analysis of skin lesions. Mod Rheumatol. 2010; 20: 123-9.
15. Brito-Zerón P, Retamozo S, Akasbi M, et al. Annular erythema in primary Sjögren's syndrome: description of 43 non-Asian cases. Lupus 2014; 23: 166-75.

16. Quartuccio L, Isola M, Baldini C, et al. Clinical and biological differences between cryoglobulinaemic and hypergammaglobulinaemic purpura in primary Sjögren's syndrome: results of a large multicentre study. Scand J Rheumatol. 2015; 44: 36-41.

17. Jennette JC, Falk RJ, Bacon PA, et al. 2012 revised International Chapel Hill Consensus Conference Nomenclature of Vasculitides. Arthritis Rheum. 2013; 65: 1-11.

18. Iaccarino L, Gatto M, Bettio S, et al. Overlap connective tissue disease syndromes. Autoimmun Rev. 2013; 12: 363-73.

19. Palm O, Garen T, Berge Enger T, et al. Clinical pulmonary involvement in primary Sjögren's syndrome: prevalence, quality of life and mortality--a retrospective study based on registry data. Rheumatology. (Oxford) 2013; 52: 173-9.

20. Yazisiz V, Arslan G, Ozbudak IH, et al. Lung involvement in patients with primary Sjögren's syndrome: what are the predictors? Rheumatol Int. 2010; 30: 1317-24.

21. Wells AU, Denton CP. Interstitial lung disease in connective tissue disease--mechanisms and management. Nat Rev Rheumatol. 2014; 10 : 728-39.

22. Stojan G, Baer AN, Danoff SK. Pulmonary manifestations of Sjögren's syndrome. Curr Allergy Asthma Rep. 2013; 13: 354-60.

23. Reina D, Roig Vilaseca D, Torrente-Segarra V, et al. Sjögren's syndrome-associated interstitial lung disease: a multicenter study. Reumatol Clin. 2016; 12: 201-5.

24. Roca F, Dominique S, Schmidt J, et al. Interstitial lung disease in primary Sjögren's syndrome. Autoimmunity Rev. 2017; 16: 48-54.

25. Ramos-Casals M, Solans R, Rosas J, et al. Primary Sjögren syndrome in Spain: clinical and immunologic expression in 1010 patients. Medicine (Baltimore). 2008; 87: 210-9.

26. Ramos-Casals M, Brito-Zerón P, Solans R, et al. Systemic involvement in primary Sjögren's syndrome evaluated by the EULAR-SS disease activity index: analysis of 921 Spanish patients (GEAS-SS Registry). Rheumatology (Oxford). 2014; 53: 321-31.

27. Goules AV, Tatouli IP, Moutsopoulos HM, Tzioufas AG. Clinically significant renal involvement in primary Sjögren's syndrome: clinical presentation and outcome. Arthritis Rheum. 2013; 65: 2945-53.

28. Ren H, Wang WM, Chen XN, et al. Renal involvement and followup of 130 patients with primary Sjögren's syndrome. J Rheumatol. 2008; 35: 278-84.

29. Maripuri S, Grande JP, Osborn TG, et al. Renal involvement in primary Sjögren's syndrome: 
a clinicopathologic study. Clin J Am Soc Nephrol. 2009; 4: 1423-31.

30. Evans R, Zdebik A, Ciurtin C, Walsh SB. Renal involvement in primary Sjögren's syndrome. Rheumatology (Oxford). 2015; 54: 1541-8.

31. Pertovaara M, Korpela M, Kouri T, Pasternack A. The occurrence of renal involvement in primary Sjögren's syndrome: a study of 78 patients. Rheumatology (Oxford). 1999; 38: 1113-20.

32. Yilmaz H, Kaya M, Özbek M, et al. Hypokalemic periodic paralysis in Sjögren's syndrome secondary to distal renal tubular acidosis. Rheumatol Int. 2013; 33: 1879-82.

33. Shavit L, Grenader T. Clinical manifestations and outcome of ANCA-related pauci-immune glomerulonephritis in patients with Sjögren's syndrome. Clin Exp Rheumatol. 2014; 32: S19-25.

34. Chai J, Logigian EL. Neurological manifestations of primary Sjögren's syndrome. Curr Opin Neurol. 2010; 23: 509-13.

35. Jamilloux Y, Magy L, Hurtevent JF, et al. Immunological profiles determine neurological involvement in Sjögren's syndrome. Eur J Intern Med. 2014; 25: 177-81.

36. Brito-Zerón P, Akasbi M, Bosch X, et al. Classification and characterisation of peripheral neuropathies in 102 patients with primary Sjögren's syndrome. Clin Exp Rheumatol. 2013; 31: 103-10.

37. Imrich R, Alevizos I, Bebris L, et al. Predominant glandular cholinergic dysautonomia in patients with primary Sjögren's syndrome. Arthritis Rheumatol. 2015; 67: 1345-52.

38. Morreale M, Marchione P, Giacomini P, et al. Neurological involvement in primary Sjögren syndrome: a focus on central nervous system. PLoS One. 2014; 9: e84605.

39. Dziadkowiak E, Sebastian A, Wiland P, et al. Endogenous event-related potentials in patients with primary Sjögren's syndrome without central nervous system involvement. Scand J Rheumatol. 2015; 44: 487-94.

40. Sheehan NJ. Dysphagia and other manifestations of oesophageal involvement in the musculoskeletal diseases. Rheumatology (Oxford). 2008; 47: 746-52.

41. Pierce JL, Tanner K, Merrill RM, et al. Swallowing disorders in Sjögren's syndrome: prevalence, risk factors, and effects on quality of life. Dysphagia. 2016; 31: 49-59.

42. Ebert EC. Gastrointestinal and hepatic manifestations of Sjögren syndrome. J Clin Gastroenterol. 2012; 46: 25-30.

43. Vassiliou VA, Moyssakis I, Boki KA, Moutsopoulos HM. Is the heart affected in primary Sjögren's syndrome? An echocardiographic study. Clin Exp Rheumatol 2008; 26: 109-12.

44. Gyöngyösi M, Pokorny G, Jambrik Z, et al. Cardiac manifestations in primary Sjögren's syndrome. Ann Rheum Dis. 1996; 55: 450-4.
45. Brito-Zerón P, Izmirly PM, Ramos-Casals M, et al. The clinical spectrum of autoimmune congenital heart block. Nat Rev Rheumatol. 2015; 11: 301-12.

46. Bartoloni E, Alunno A, Bistoni O, et al. Characterization of circulating endothelial microparticles and endothelial progenitor cells in primary Sjögren's syndrome: new markers of chronic endothelial damage? Rheumatology (Oxford). 2015; 54: 536-44.

47. Atzeni F, Sarzi-Puttini P, Signorello MC, et al. New parameters for identifying subclinical atherosclerosis in patients with primary Sjögren's syndrome: a pilot study. Clin Exp Rheumatol. 2014; 32: 361-8.

48. Sabio JM, Sánchez-Berná I, Martinez-Bordonado J, et al. Prevalence of and factors associated with increased arterial stiffness in patients with primary Sjögren's syndrome. Arthritis Care Res (Hoboken). 2015; 67: 554-62.

49. Ciçek OF, Bayram NA, Ayhan H, et al. Assessment of the relationship between aortic stiffness and left ventricular functions with echocardiography in patients with Sjögren's syndrome. Int J Rheum Dis. 2014; 17: 658-63.

50. Gravani F, Papadaki I, Antypa E, et al. Subclinical atherosclerosis and impaired bone health in patients with primary Sjögren's syndrome: prevalence, clinical and laboratory associations. Arthritis Res Ther. 2015; 17: 99.

51. Bartoloni E, Baldini C, Schillaci G, et al. Cardiovascular disease risk burden in primary Sjögren's syndrome: results of a populationbased multicentre cohort study. J Intern Med. 2015; 278: 185-92.

52. Juarez M, Toms TE, de Pablo P, et al. Cardiovascular risk factors in women with primary Sjögren's syndrome: United Kingdom primary Sjögren's syndrome registry results. Arthritis Care Res (Hoboken). 2014; 66: 757-64.

53. Augusto KL, Bonfa E, Pereira RM, et al. Metabolic syndrome in Sjögren's syndrome patients: a relevant concern for clinical monitoring. Clin Rheumatol. 2016; 35: 639-47.

54. Ramos-Casals M, Font J, Garcia-Carrasco M, et al. Primary Sjögren syndrome: hematologic patterns of disease expression. Medicine (Baltimore). 2002; 81: 281-92.

55. Fox RI. Sjögren's syndrome. Lancet 2005; 366 : 321-31.

56. Koh JH, Lee J, Chung SH, et al. Relation of autoimmune cytopenia to glandular and systemic manifestations in primary Sjögren syndrome: analysis of 113 Korean patients. J Rheumatol. 2015; 42: 1817-24.

57. Quartuccio L, Baldini C, Priori R, et al. Cryoglobulinemia in Sjögren syndrome: a disease subset that links higher systemic disease activity, autoimmunity, and local B cell proliferation in mucosa-associated lymphoid tissue. J Rheumatol 2017 [Epub ahead of print]. 